\title{
IDENTIFICATION OF ELASTIC CONSTANTS OF THE REINFORCED ORTHOTROPIC COMPOSITE GROUND FROM FIELD MEASUREMENTS
}

\author{
Hyu-Soung Shin \\ Geotechnical Engineering Research Dept. \\ Korea Institute of Construction Technology \\ 2311 Daehwa, ilsan, Goyang, Gyeonggi, South Korea \\ hyushin@kict.re.kr
}

\author{
Gyan N. Pande \\ Centre for Civil and Computational Engineering \\ Singleton Park, Swansea, SA2 8PP, UK \\ g.n.pande@swansea.ac.uk
}

\begin{abstract}
In this paper, a new methodology for identifying numerous elastic parameters of an orthotropic ground material from field measurements is presented. For a better understanding, a simpler problem, which, however, is in the same concept, is adopted for describing the strategy, which is to identify an orthotropic material from a single structural test. At the heart of the methodology is the self-learning algorithm that is to extract various stress-strain relationships from a single structural test and train a neural network with the relationships in finite element framework. The constitutive matrix resulting from the trained neural network based constitutive model (NNCM) is compared with the conventional constitutive matrix for an orthotropic material to determine the nine independent elastic constants. An example is given for better understanding of the methodology proposed.
\end{abstract}

Keywords: Neural Network, Identification, Orthotropic Material, Field Measurement, Tunnel

\section{Introduction}

The state of stresses in the monitored ground around an excavation site (ground material with non-uniform stress field) is admittedly complex and different points in it follow widely different stress paths. However, it has recently been shown that data of displacements measured at several points on the excavated surface can be used to train a neural network embedded in a finite element code which replaces a conventional constitutive model. The constitutive matrix resulting from the trained neural network is compared with the conventional constitutive matrix for an orthotropic ground material to determine the nine independent elastic constants.

In this paper, the authors demonstrate a new methodology in which the orthotropic elastic parameters can be obtained by monitoring displacement induced by applying a load on the structure at a convenient location. This is a classical 'inverse' problem, which in the past has been solved by many researchers using conventional back analyses for the identification of elastic and strength parameters for isotropic materials [1-3]. However, it is not a simple extension of known procedures if parameters for an anisotropic or even an orthotropic material were to be identified. There have been a number of applications of tools of artificial intelligence for the identification of material parameters from the case histories of monitored data for structures as well as tunnels using neural networks $[4,5]$. However, the assumption of isotropy is inherent in these applications.

In this paper, a new two-step methodology is proposed in which, in the first step, the monitored data of a structure are used to recursively train a neural network based constitutive model (NNCM) embedded in a finite element code and in the second step, the required material parameters are computed from the trained NNCM through 'virtual' tests on specimens having appropriate boundary and loading conditions. Although the example given is that of linear orthotropic material, the procedure is generic and applicable to anisotropic nonlinear elastic materials as well.

\section{Methodology of identification of parameters from a trained NNCM}

2.1 Methodology of identification of parameters from a trained NNCM

Numerous applications of tools of artificial intelligence to engineering problems are currently being reported by various researchers. A neural network attempts to find a pattern between 'causes' and 'effects'. If we consider an application to material models in the context of finite element modelling, the components of strain become 'causes' whilst resulting stresses are deemed as the 'effects'. Strain and stress vectors,

$$
\begin{aligned}
& \boldsymbol{\varepsilon}_{i}=\left\{\varepsilon_{1}, \varepsilon_{2}, \varepsilon_{3}, \gamma_{12}, \gamma_{23}, \gamma_{31}\right\}, \\
& \sigma_{k}=\left\{\sigma_{1}, \sigma_{2}, \sigma_{3}, \tau_{12}, \tau_{23}, \tau_{31}\right\}
\end{aligned}
$$

are introduced as input nodes, $o_{i}$ and output nodes, $o_{k}$ of the NN, respectively. Then, in the neural network nomenclature, stress-strain relation can be represented as

$$
\sigma_{k}=N N\left(\varepsilon_{i}\right) .
$$

The optimal architecture of the NN adopted is shown in Fig. 1. It has 6 nodes each in input and output layers. There are two hidden layers with 12 nodes in each of the layers. Thus, the neural network can be designated as $\mathrm{NN}(6-12-12-6)$. The first derivative of the $\mathrm{NN}, D N N_{i k}$ 
becomes the so called 'tangential stiffness' matrix for a material at a certain given strain state, $\boldsymbol{\varepsilon}_{i}$. It can be written as follows:

$$
D_{N N}=D N N_{i k}\left(\varepsilon_{i}, \sigma_{k}\right)=\frac{\partial \sigma_{k}}{\partial \varepsilon_{i}} .
$$

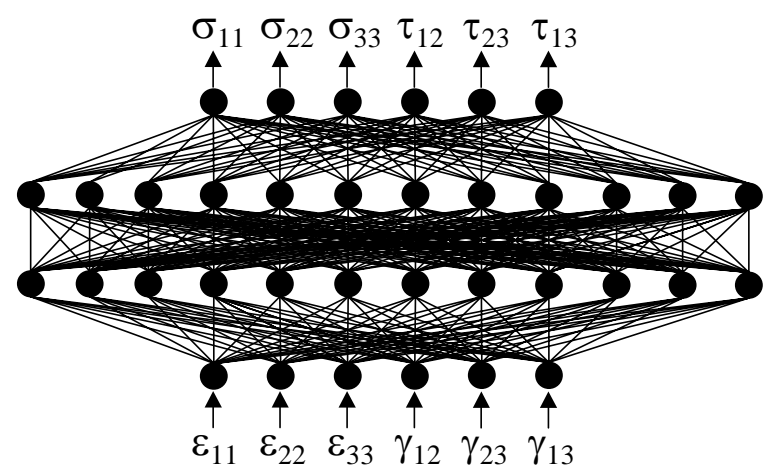

Figure 1. Optimal architecture of the NNCM.

A formulation to compute accurately the first derivative of a NN has recently been developed by the authors [6,7]. Eq. 3 can be applied in both linear and nonlinear range. NNCMs do not invoke a-priori any mathematical constitutive framework. There is also no assumption of isotropy. Therefore, once a NN has been trained for a material, stresses corresponding to any level of strains can be readily computed from Eq. 2. Moreover, a derivative, $D_{N N}$ can be composed at the same level of strain using Eq. 3 . The constitutive matrix resulting from the NNCM can then be directly incorporated in a conventional FE analysis code in place of the conventional mathematical theory based constitutive models. Furthermore, with the NN based constitutive matrix it becomes possible to use a NNCM in incremental form of stresses for structural analysis although the NNCM may have initially been trained with the data in the form of total strains and corresponding stresses.

The size of $D_{N N}$ for three-dimensional analysis is the same as a normal constitutive matrix (i.e. $6 \times 6$ ). The computed $D_{N N}$ is generally non-symmetric. It is symmetrised by averaging the off diagonal terms. Comparison of each component of the general elasticity matrix with the computed $D_{N N}$ is made to set up a system of simultaneous equations. This leads to identification of 21 anisotropic independent constants for the general case and nine constants (i.e. $E_{x}, E_{y}, E_{z}, v_{x y}, v_{y z}, v_{x z}, G_{x y}, G_{y z}, G_{x z}$ ) for the orthotropic case.

2.2 Training of a NNCM from monitored data of load versus displacement

A NNCM can obviously be trained with the experimental data of stress versus strain. It has been recently demonstrated by Ghaboussi et al. (1998) and Shin and Pande (2000) that NNCMs can also be trained through incremental load-displacement data monitored at several points on the structure; many or all of these points may well be on the surface or the boundary for convenience. Here, a NNCM is embedded in a finite element code and a nonlinear incremental analysis of the boundary value problem is carried out using a 'pre-primed' NNCM (an arbitrary linear tangential stiffness matrix, for example). This gives the computed displacement field from which a vector of displacements for the monitored points $\left(\delta_{c}^{n}\right)$ can be assembled. This would obviously differ from the displacements observed since an arbitrary constitutive matrix was adopted to start the iterative process. In the following step, the same boundary value problem is re-solved in which discrepancy in measured and computed displacements is applied as prescribed displacements. The stresses and strains at each Gauss point and at each increment constitute a set of data for training of the NNCM. The NNCM is recursively trained until the an indicator of discrepancy between predicted and monitored displacement fields, $\xi$, defined by

$$
\xi=\frac{1}{l \times k} \sum_{n=1}^{l} \sum_{i=1}^{k}\left(\left|\delta_{m}^{i}-\delta_{c}^{i}\right| /\left|\delta_{m}^{i}\right|\right)_{n}
$$

is less than a prescribed acceptable value. In the above equation, $l$ and $k$ are the number of total load increments and monitoring points, respectively. And $\delta_{m}^{i}$ and $\delta_{c}^{i}$ are monitored and calculated displacements at ith monitoring points, respectively. Thus, at the end of the iterative process, the constitutive model embedded in the FE code is such that it predicts the monitored displacements. A flow diagram of the computational algorithm can be found in [6]. The finite element code which has a NNCM embedded in it has been termed by the authors as a 'self-learning' code since it has the capability to update its constitutive model. If there are sufficient number of strategically placed monitoring points, it can be assumed that the NNCM is sufficiently trained to predict stress-strain response under arbitrary stress paths. A derivative of the trained NNCM, leads to the evaluation of linear or nonlinear elastic constants at any strain level.

\section{An illustrative example}

For heuristic purposes, a simple structure viz. a plane stress panel with a circular hole at its contre is chosen. The panel which is assumed to be made of an orthotropic material with known principal material axes. Fig. 2 shows the geometry, boundary and loading conditions. It is well known that severe stress concentrations take place around the cavity. A number of monitoring points at convenient locations have to be chosen. It is obvious that larger the number of monitoring points more accurate stress-strain relation can be extracted from the NNCM. Although, collecting data for a large number of monitoring points may lead to higher costs, it should be noted that modern instrumentation techniques with automatic data-loggers make such field measurements relatively easy to conduct. 
The authors, at this stage, did not wish to divert their attention to a field measurement programme to obtain monitored displacement data. It was therefore decided to carry out a conventional 3-D FE analysis of the panel configuration using assumed values of the nine independent orthotropic elastic constants as given in Table 1. A three-dimensional analysis, instead of a two dimensional plane stress analysis, was intentionally carried out so as to prove that the methodology is generic and elastic parameters can be identified from displacement - load data of arbitrary shaped bodies. A quarter symmetric model was discretised with 578 nodes and 70 isoparametric 20 noded brick elements. It is noted that the accuracy of the FE model adopted is not an issue here.

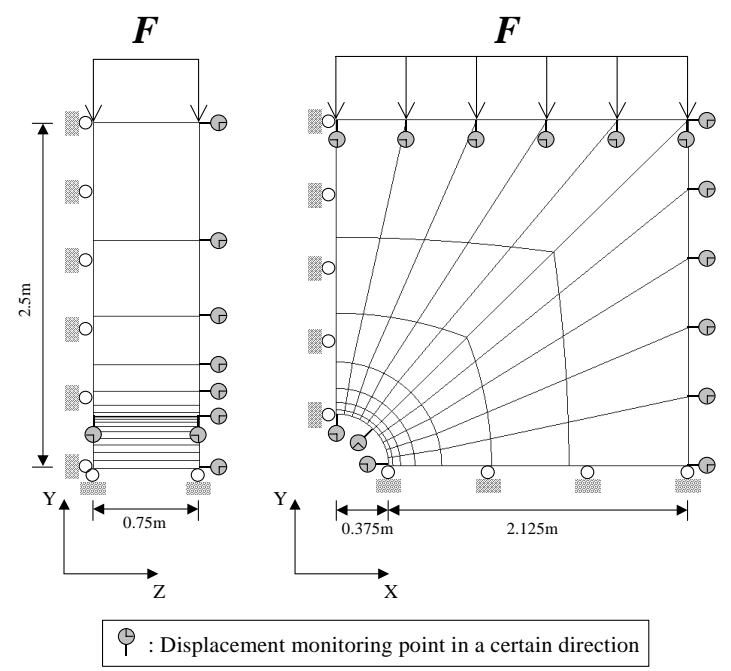

Figure 2. A panel with circular cavity at its centre.

Displacements of 66 nodes on the surface of the model at 5 load levels, resulting from the FE analysis were assumed as physically measured data from the field test, although a much smaller number of monitoring points would be adequate as shown by Shin and Pande (2000). These are the only data needed for training the NNCM embedded in the self-learning FE code. It is noted that although the material is linear with proportional load-displacement relationship, the NNCM cannot recognise this unless incremental data are given.

As mentioned in the previous section, some initial data are required for 'priming' the NNCM. In this example, the values of isotropic elastic constants, $E=1.0 \mathrm{GPa}$ and $v=0.2$ were arbitrarily adopted for priming. At the $3^{\text {rd }}$ cycle of self-learning process, the overall discrepancy between predicted and monitored displacement field reduced from $37.21 \%$ to $2.76 \%$.

Table 1. Arbitrarily chosen orthotropic elastic constants.

\begin{tabular}{cc}
\hline Elastic constants & Assumed values \\
\hline $\mathrm{E}_{\mathrm{x}}(\mathrm{MPa})$ & 650.4 \\
$\mathrm{E}_{\mathrm{y}}(\mathrm{MPa})$ & 967 \\
$\mathrm{E}_{\mathrm{z}}(\mathrm{MPa})$ & 1735.4 \\
$\mathrm{G}_{\mathrm{xy}}(\mathrm{MPa})$ & 582.2 \\
$\mathrm{G}_{\mathrm{yz}}(\mathrm{MPa})$ & 203.6 \\
$\mathrm{G}_{\mathrm{xz}}(\mathrm{MPa})$ & 694.5 \\
$v_{\mathrm{xy}}$ & 0.269 \\
$v_{\mathrm{yz}}$ & 0.139 \\
$v_{\mathrm{xz}}$ & 0.13 \\
\hline
\end{tabular}

\subsection{Identification of orthotropic elastic constants}

At the beginning of each iteration of the self-learning FE analysis, constitutive matrices at all Gauss points are computed using Eq. 3. These are compared to the form of the conventional orthotropic elasticity matrix as the material assumed for this example is an orthotropic material with material axis coinciding with the axis of the model. The elastic constants are simply determined by forming a set of equations with elastic parameters as unknowns. It is noted that NN based constitutive matrices do not invoke any symmetry and as many as 36 elastic constants can be evaluated.

The elastic constants are evaluated at the middle of each

Table 2. Prediction of orthotropic elastic constants after each self-learning process.

\begin{tabular}{|c|c|c|c|}
\hline Ratios & $\begin{array}{c}\text { Prediction after } 1^{\text {st }} \\
\text { self-learning }\end{array}$ & $\begin{array}{c}\text { Prediction after } 2^{\text {nd }} \\
\text { self-learning }\end{array}$ & $\begin{array}{c}\text { Prediction after } 3^{\text {rd }} \\
\text { self-learning }\end{array}$ \\
\hline $\mathrm{E}_{\mathrm{x}, \mathrm{p}} / \mathrm{E}_{\mathrm{x}, \mathrm{a}}$ & $103.4 \%$ & $100.06 \%$ & $100.24 \%$ \\
\hline $\mathrm{E}_{\mathrm{y}, \mathrm{p}} / \mathrm{E}_{\mathrm{y}, \mathrm{a}}$ & $75.67 \%$ & $98.55 \%$ & $99.02 \%$ \\
\hline $\mathrm{E}_{\mathrm{z}, \mathrm{p}} / \mathrm{E}_{\mathrm{z}, \mathrm{a}}$ & $65.39 \%$ & $74.63 \%$ & $103.46 \%$ \\
\hline$G_{x y, p} / G_{x y, a}$ & $42.82 \%$ & $69.36 \%$ & $101.53 \%$ \\
\hline $\mathrm{G}_{\mathrm{yz}, \mathrm{p}} / \mathrm{G}_{\mathrm{yz}, \mathrm{a}}$ & $108.4 \%$ & $106.1 \%$ & $99.95 \%$ \\
\hline$G_{x z, p} / G_{x z, a}$ & $64.78 \%$ & $67.86 \%$ & $102.95 \%$ \\
\hline$v_{x y, p} / v_{x y, a}$ & $72.86 \%$ & $99.63 \%$ & $101.49 \%$ \\
\hline$v_{\mathrm{yz}, \mathrm{p}} / \mathrm{v}_{\mathrm{yz}, \mathrm{a}}$ & $25.9 \%$ & $83.45 \%$ & $99.28 \%$ \\
\hline$v_{\mathrm{xz}, \mathrm{p}} / v_{\mathrm{xz}, \mathrm{a}}$ & $111.54 \%$ & $113.85 \%$ & $96.15 \%$ \\
\hline$\xi(\%)$ & $37.21 \%$ & $13.93 \%$ & $2.76 \%$ \\
\hline
\end{tabular}


load increments of each cycle of the self-learning procedure. Table 2 shows the evolution of nine orthotropic elastic constants with the number of cycles of re-training of the NNCM embedded in the FE code. At the $3^{\text {rd }}$ cycle of self-learning process all the values of the orthotropic constants were less than $3.8 \%$ in error against the target values. It is found from numerical experiments that in practical problems tested so far no more than five self-learning cycles are required.

\section{Summary and conclusions}

In this study, a methodology to identify anisotropic material parameters from a single structural test has been proposed. First, a NNCM embedded in a FE code was trained from the load displacement data of a structural test. In the absence of real load displacement data, load displacement data from a FE analysis based on an assumed orthotropic elastic material model were used. After the $3^{\text {rd }}$ cycle of re-training of the NNCM the nine elastic parameters of the orthotropic material were computed with the maximum error in any parameter being less than $4 \%$.

The methodology of parameter identification for anisotropic elastic constants proposed here is valid for fully anisotropic materials having 36 constants although the example of an orthotropic material has been illustrated in this paper. Although, a rather simple test configuration of plane stress panel with a central cavity was used for illustration, the methodology is generic and elastic constants can be identified from instrumented load-displacement data of three-dimensional bodies of arbitrary shape. A procedure for identifying strength parameters as well as elastic constants from a single structural test is currently being investigated. This may lead to a novel method of non-destructive testing.

\section{REFERENCES}

[1] Gioda G, Maier G., “Direct search solution of an inverse problem in elastoplasticity: Identification of cohesion, friction angle and in situ stress by pressure tunnel tests", Int. J. Numer. Meth. Engng., Vol. 15, pp. 1823-1848, 1980.

[2] Sakurai S, Takeuchi K., "Back analysis of measured displacements of tunnels", Rock Mechanics and Rock Engineering, Vol. 16, pp. 173-180, 1983.

[3] Ohkami T, Swoboda G. "Parameter identification of viscoelastic materials" Computers and Geotechnics. Vol. 24, pp. 279-295, 1999.

[4] Fairbairn EMR, Ebecken NFF, Paz CNM, Ulm F-J., "Determination of probabilistic parameters of concrete: solving the inverse problem by using artificial neural networks", Computers \& Structures, Vol. 78, pp. 497-503, 2000.

[5] Pichler B, Lackner R, Mang HA., "Soft-computing based structural back analysis of material model parameters for soil in the context of tunnelling according to the new Austrian tunnelling method”, In:
Valliappan S and Khalili N, editors. Proc. Asian Pacific Computational Mechanics -New frontiers for new millennium. Elsevier Science Ltd., pp. 1101-1108. 2001.

[6] Shin H. S. Neural network based material models for finite element analysis, Ph.D. thesis: $\mathrm{C} / \mathrm{Ph} / 250 / 01$. Department of Civil Engineering, University of Wales Swansea, November 2001.

[7] Shin H.S, Pande G.N., "Intelligent finite elements", In: Valliappan S and Khalili N, editors, Proc. Asian Pacific Computational Mechanics -New frontiers for new millennium, Elsevier Science Ltd., pp. 1301-1310, 2001

[8] Ghaboussi J, Pecknold D.A., Zhang M, Haj-ali R.M., "Autoprogressive training of neural network constitutive models”, Int. J. Numer. Meth. Engng, Vol. 42, pp. 105-126, 1998.

[9] Shin H.S, Pande G.N. "On self-learning finite element code based on monitored response of structures". Computers and Geotechnics, Vol. 27, pp. 161-178, 2000.

[10] Rumelhart DE, McClelland JL. Parallel Distributed Processing Vol. 1\&2, Massachusetts: The MIT Press, 1988.

[11] Pao Y.H., Adaptive pattern recognition and neural networks. Addison-Wesley Publishing Company Inc, 1989. 